Domain structure in polycrystalline $\mathrm{MnZn}$ ferrite imaged by magnetic force microscopy

J. Aarts and I. Abu ShiekahP. J. van der Zaag

Citation: 85, (1999); doi: 10.1063/1.369353

View online: http://dx.doi.org/10.1063/1.369353

View Table of Contents: http://aip.scitation.org/toc/jap/85/10

Published by the American Institute of Physics 


\title{
Domain structure in polycrystalline MnZn ferrite imaged by magnetic force microscopy
}

\author{
J. Aarts ${ }^{\text {a) }}$ and I. Abu Shiekah \\ Kamerlingh Onnes Laboratory, Leiden University, P.O. Box 9504, 2300 RA Leiden, The Netherlands \\ P. J. van der Zaag \\ Philips Research Laboratories, Prof.Holstlaan 4, 5656 AA Eindhoven, The Netherlands
}

(Received 11 May 1998; accepted for publication 24 January 1999)

\begin{abstract}
We investigated the occurrence of magnetic domains in MnZn-ferrite grains by magnetic force microscopy (MFM). At the surface of samples with different grain sizes in the range from 2 to 10 $\mu \mathrm{m}$, small grains generally contain only one domain, while large grains contain two domains. The crossover between these two intragranular domain states occurs at a critical grain size $D_{\text {cr }}$ of about $4 \mu \mathrm{m}$. This finding is in agreement with the conclusions from earlier neutron depolarization measurements on the same samples and reemphasizes the large discrepancy between theoretical calculations of $D_{\text {cr }}$ for isolated grains and experimental values in the ferrites. Furthermore, we find that the magnetic field profiles are not those expected from isolated grains. Modeling of the MFM response shows that the magnetic environment of a grain plays an important role in its magnetic configuration. (C) 1999 American Institute of Physics. [S0021-8979(99)02709-7]
\end{abstract}

\section{INTRODUCTION}

Knowledge of the domain structure in a magnetic material is a key factor in understanding its magnetic response. An important class of magnetic materials is formed by soft magnetic ferrites with the Spinel-type crystal structure. Due to their low conductivity they are used for high frequency applications $(f>10 \mathrm{kHz})$. For optimum performance at these high frequencies also their magnetic hysteresis or dissipation should be as low as possible. ${ }^{1,2}$ Since this property is related to domain wall motion, several studies have been performed lately on the domain structure of poly-crystalline MnZn and $\mathrm{NiZn}$ ferrites, and its relation to the average grain size. ${ }^{3-5}$ The latter parameter can be controlled by the sinter temperature in the preparation process and allows tuning of the grain size with small size distributions from below $1 \mu \mathrm{m}$ to above $10 \mu \mathrm{m}$ while maintaining a constant composition. ${ }^{6,7}$ Due to the low magneto-crystalline anisotropy of certain MnZnferrite compositions, the domain wall size can be very large, of the order of $1 \mu \mathrm{m}$, so that the grain size can be varied from below to above the domain wall size. This has several consequences. For instance, it was shown by neutron depolarization (ND) studies that a critical grain size $D_{\text {cr }}$ exists, below which the grains are mono-domain. ${ }^{3,4}$ For the $\mathrm{MnZn}$ ferrites to be discussed here, $D_{\text {cr }}$ is about $4 \mu \mathrm{m}$. Recently, it was found from magnetic susceptibility measurements that the energy dissipation at $\mathrm{MHz}$ frequencies is substantially lower in samples with grain size $D<D_{\text {cr }}$, which was attributed to the absence of domain walls in these small grains. ${ }^{5,8}$

Still, a number of questions persists. For instance, theoretical estimates for $D_{\text {cr }}$ of an isolated grain ${ }^{9-12}$ yield values which are orders of magnitude lower than experimentally found. ${ }^{4,10}$ Even when taking into account that the grain is embedded in a soft magnetic environment, the upper limit

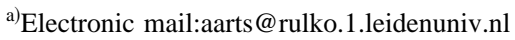

for the estimates of $D_{\mathrm{cr}}$ barely reaches the experimental value. ${ }^{5,12}$ Moreover, the change in energy dissipation as function of grain size can be surprisingly sharp, taking place in less than $0.1 \mu \mathrm{m} .{ }^{5}$ Given the distribution of grain sizes inherent to ceramic materials, the expectation would be a more gradual change in the region where grains with single domains and two domains should coexist. Better understanding is clearly needed of the behavior of the magnetization and the domain wall in a grain in connection with its local environment. Moreover, since the data from ND and dissipation measurements reflect the average response of the bulk material, it seems desirable to supplement the results of these experiments with microscopic information on the level of single grains.

In principle, magnetic force microscopy (MFM) can provide information on the existence of domains and domain walls. The main question to be addressed in this article will be whether, in grains on the sample surface, a similar connection can be made between grain size and number of domains as found from the bulk-averaging ND measurements, using the same MnZn-ferrite samples as previously studied by neutron depolarization.

The article is arranged as follows; since the glaring discrepancies between measured and calculated values of $D_{\text {cr }}$ are the main motivation for our investigation, we first recapulate the basis of such calculations in Sec. II. Section III deals with the experimental details. In Sec. IV we present the data. They show that the samples with small grains do not show magnetic contrast, which we interpret as the absence of domain walls. In large-grained samples, magnetic contrast c.q. a domain wall is always present within the grain, with a crossover regime from $D=3 \mu \mathrm{m}$ to $D=5 \mu \mathrm{m}$. These numbers are essentially equivalent to the value $D_{\mathrm{cr}}=4 \mu \mathrm{m}$ found in the ND measurements. Apart from this main conclusion, we find that the large grains show two different types of 
magnetic contrast. An Appendix is added to discuss this observation. Although the issue cannot be fully resolved, simple model calculations indicate that such behavior is not expected for isolated grains. Apparently, neighboring grains play a role in determining the distribution of the surface magnetization and therefore, possibly in the domain wall formation.

\section{THEORETICAL ESTIMATES OF $D_{\text {cr }}$}

Theoretically, for an isolated magnetic particle a transition to a monodomain state of uniform magnetization is expected with decreasing grain size, since the domain wall energy scales with the domain wall area, approximately as $D^{2}$, whereas the magnetostatic energy decreases more rapidly with $D^{3} \cdot{ }^{10,13}$ On a more quantitative level, exact expressions were derived by Brown for the lower and upper bounds of the critical radius of a spherical particle. ${ }^{9}$ There are two fundamental complications in comparing an experimentally determined $D_{\text {cr }}$ to a theoretically expected value. One is that the particle is usually not spherical, so that the magnetization need not be uniform and the nucleation process for a domain wall can be different. The other is that the micromagnetic theory ${ }^{9,13}$ deals with isolated grains. In the bulk ferrite discussed here, the grains are neither spherical not isolated and especially the soft-magnetic environment of the grain should be taken into account. ${ }^{4,14}$ This yields correlations between the magnetization direction in neighboring grains such that the condition $\operatorname{div} M \approx 0$ between such grains is met (for instance, in the case when four neighboring grains form a diamond-like magnetization structure). In a first approximation for the calculation of $D_{\mathrm{cr}}$, one can study the effect of the soft-magnetic environment by (artificially) lowering the magnetostatic energy of the grain. ${ }^{14}$ This can be done either by using a reduced effective value for the saturation magnetization $M_{s},{ }^{12}$ or by introducing an (isotropic) initial permeability. ${ }^{4}$ The latter yields a reduction of the demagnetization energy by a factor $\frac{2}{3} \mu_{i}$. Applying the permeability correction to the upper and lower limits for the critical grain size derived by Brown ${ }^{9}$ yields

$$
\begin{aligned}
& D_{\text {cr,lower }}=3.6 \sqrt{\frac{\frac{2}{3} A}{\frac{1}{2} \mu_{0} M_{S}^{2}}}, \\
& D_{\text {cr,upper }}=28 \sqrt{\frac{\frac{2}{3} A}{\frac{1}{2} \mu_{0} M_{S}^{2}}} \sqrt{\pi+\frac{\frac{2}{3} \mu_{i} K}{\frac{1}{2} \mu_{0} M_{S}^{2}}},
\end{aligned}
$$

with $A$ the exchange constant, $\mu_{i}$ the initial permeability at $D_{\text {cr }}, \mu_{0}$ the vacuum permeability and $K$ the anisotropy constant. Using the numerical values pertaining to the $\mathrm{MnZn}$ ferrite studied here, ${ }^{4} A=3 \times 10^{-12} \mathrm{~J} / \mathrm{m}, \quad \mu_{i}=1200, \quad K$ $=32 \mathrm{~J} / \mathrm{m}^{3}$, and $\mu_{0} M_{s}=0.52 \mathrm{~T}$ yields a lower limit of 0.54 $\mu \mathrm{m}$ and an upper limit of $7.7 \mu \mathrm{m}$ for $D_{\text {cr }}$, in quite reasonable agreement with the experimentally found $D_{\mathrm{cr}} \approx 3.8 \pm 0.7 \mu \mathrm{m}$, by neutron depolarization. ${ }^{4}$ Note that the soft-magnetic environment correction has increased the calculated critical grain size by three orders of magnitude. The other approach to the problem, of reducing $M_{s}$, was applied recently in a numerical computation of $D_{\text {cr }}$ in NiZn ferrite. Using the bulk value for $M_{s}$, the computation yielded $D_{\mathrm{cr}} \approx 22 \mathrm{~nm}$; a reduction of $M_{s}$ (i.e., the magnetostatic energy of the grain) by a factor of 20 was needed to come close to the experimental value of $1.4 \mu \mathrm{m} .{ }^{12}$ Summarizing, major corrections are needed to reproduce the experimental (ND) values for $D_{\mathrm{cr}}$ in ferrites. An independent determination of these values will therefore be useful.

\section{EXPERIMENT}

The investigated MnZn ferrites were the same as used in the earlier studies cited earlier. ${ }^{3,4}$ Their composition is $\mathrm{Mn}_{0.60} \mathrm{Zn}_{0.35} \mathrm{Fe}_{2.05} \mathrm{O}_{4}$. They were prepared by using wet chemical methods in the powder preparation and using sinter temperatures between 750 and $1250^{\circ} \mathrm{C}$ which yields grain sizes between 0.2 and $16 \mu \mathrm{m} .{ }^{6,7}$ As a measure for the grain size $D$, the mean linear intercept was taken as determined from electron microscopy images. For this study, samples with four different grain sizes around the value for $D_{\text {cr }}$ (known from the ND experiments) were selected from the samples previously investigated by ND: $2.5,3.1,5.4$, and 7.3 $\mu \mathrm{m}$. For briefness, they will be designated as MZ25, MZ31, MZ54, and MZ73. The samples were embedded in epoxy and polished using Seyton ${ }^{(r)}$ to flat and shiny surfaces for the MFM measurements.

The MFM used employs a home-built piezo-tube scanner with a lateral range of $10 \mu \mathrm{m}$ on which the sample is mounted, commercial atomic force microscopy triangular cantilevers with gold coating for enhanced reflectivity at the back, and optical detection of the deflection by using a laser diode and a position sensitive detector. Scan control and data acquisition were performed by commercial electronics (RHK Inc.). The $\mathrm{Si}_{3} \mathrm{~N}_{4}$ tips were made magnetically sensitive by evaporation of a thin Fe film of about $80 \mathrm{~nm}$, and covering this with $20 \mathrm{~nm}$ Au for oxidation protection. A shadow mask was used during the evaporation, in order to prevent coating of the cantilever arms. All experiments were performed at room temperature, as were the previous (ND) experiments. ${ }^{3,4}$ Surface topology of the samples was measured in contact mode. The magnetic response of single grains was measured in two steps. First, the grain was scanned in contact mode and a tilt compensation was made by mixing the lateral $(X, Y)$ scan voltages with the height $(Z)$ scan voltage. Subsequently, with the scan plane now equivalent to the plane of the grain, the tip was retracted about $100 \mathrm{~nm}$. This is relatively far from the surface, but that was necessitated by the relatively large height differences on especially the fine grained samples, as well as by the sometimes large magnetic signals near grain boundaries and pores. The magnetic signal was measured in dynamic mode. The cantilever, which had a force constant of $0.1 \mathrm{~N} / \mathrm{m}$, was driven to oscillate with a frequency close to its resonance frequency, around $30 \mathrm{kHz}$, and an amplitude of about $10 \mathrm{~nm}$. Changes in the resonance frequency of the oscillating cantilever were detected with a lock-in amplifier by measuring the changes in vibration amplitude. Thus, actually the derivative of the force on the cantilever in the direction along the tip ${ }^{15}$ is measured. Images were usually taken along 256 or 512 lines, with 256 (512) points per line. For a clear representation of the different 


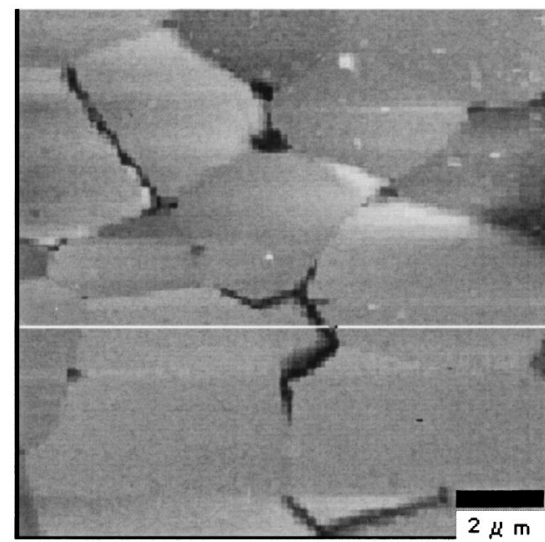

FIG. 1. Topographic image of sample MZ25, with an average grain size of $2.3 \mu \mathrm{m}$. The gray scale corresponds to maximum height differences of about $200 \mathrm{~nm}$. The marker has a width of $2 \mu \mathrm{m}$.

features in the images, signal changes will be shown along cross-sectional lines, which were obtained as follows. In topographical images, one image scan line could be taken directly, without further treatment. In the magnetic images, improvement of signal-to-noise ratio was needed. In a scanned line, a smoothing procedure was performed over 15 adjacent points, which typically corresponds to a width, and therefore a resolution, of about $0.3 \mu \mathrm{m}$. Five such lines (typical distance $0.02 \mu \mathrm{m}$ ) were then averaged.

\section{RESULTS ON THE DOMAIN STRUCTURE}

In this section, topographic and magnetic images are presented from samples with different grain sizes. Magnetic contrast is found within large grains and interpreted as the occurrence of two magnetic domains.

In Fig. 1, a topographic image of $12 \times 12 \mu \mathrm{m}$ is shown for sample MZ25. A number of features can be seen. First, there is a clear distribution in grain sizes. The diameter of the smallest grain in the images is about $2 \mu \mathrm{m}$; the diameter of the largest grain is over $4 \mu \mathrm{m}$. This is not in contradiction with the quoted average grain size, which was defined as the mean chord along an arbitrary line in an electron micrograph ${ }^{4}$ and determined from averaging lines in several pictures taken at various parts of the sample. Second, the outlines of the grains can be seen clearly. The contrast only derives from height differences, indicating that the polishing is more effective for some grains than for others. ${ }^{16}$ For MZ25, the maximum step height found was about $200 \mathrm{~nm}$. Finally, pores are also clearly present on the surface. Figure 2 shows a topographic image and a magnetic image of one selected grain in MZ25 with dimensions of about $4 \times 4 \mu \mathrm{m}$. In the magnetic image, the outline of the grain is reproduced as a thin black line, since it is difficult to detect in this image. Two cross sections were made in both images, left-to-right and bottom-to-top, shown as thin white lines. We designate them HT, HM (horizontal topographic, magnetic) and VT, VM (vertical topographic, magnetic). The data are given in Fig. 3. The HT line [dotted line in Fig. 3(a)] shows a flat surface with steps at both grain boundaries. In the HM line there is a corresponding change in signal level at the right-

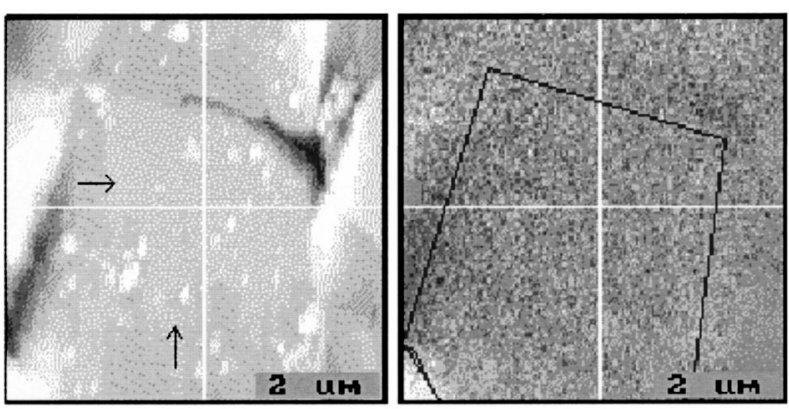

FIG. 2. Images of a single grain in sample MZ25. (Left) topographic image; (right) magnetic image. The markers have a width of $2 \mu \mathrm{m}$. The white lines show the cross sections given in Fig. 3 (with directions as indicated by the arrows). The outline of the grain in the magnetic image is shown in black.

hand grain boundary, but otherwise it is rather featureless. The VT line only crosses one grain boundary with a somewhat smaller step. The corresponding VM line does not show any feature. The small-scale oscillations are due to the smoothing-averaging procedure; the period is of the order of the resolution, the height is representative of the residual noise. Note that for this grain, apparently, the surrounding material has the same direction of the magnetization. Otherwise, the ensuing stray fields would have led to much stronger signal variations near the grain boundary.
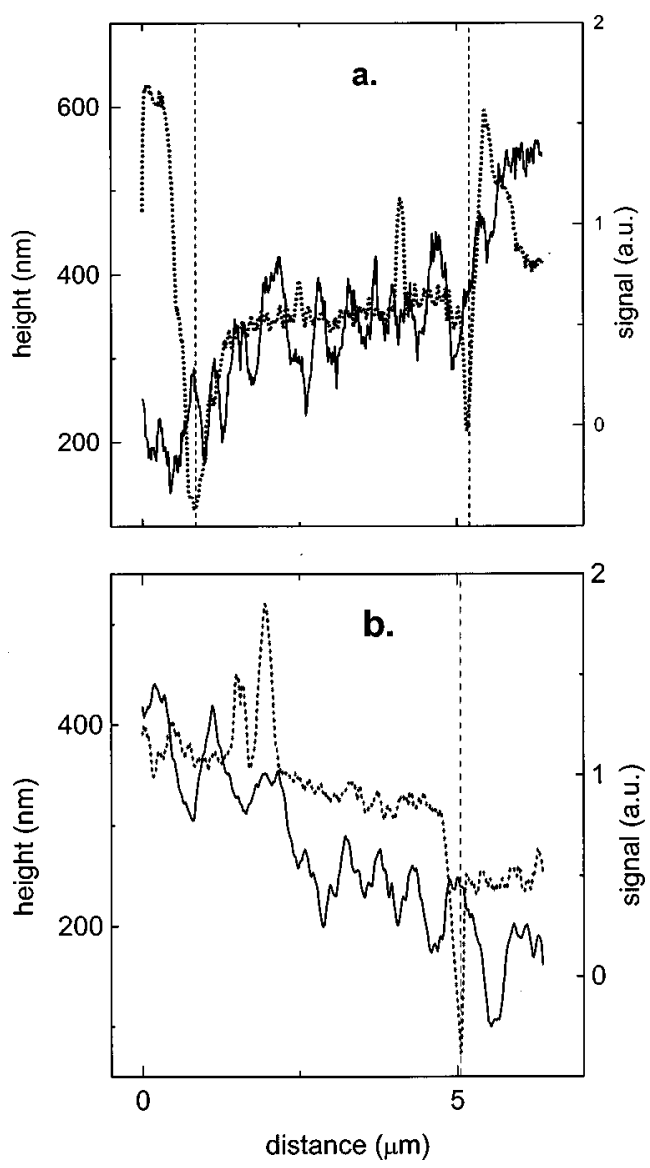

FIG. 3. Topographic (dotted line; left-hand scale) and magnetic (drawn line; right hand scale) signal variations along the cross-sectional lines given in Fig. 2. The dashed vertical lines indicate the grain boundaries. (a) Left-toright (called HT and HM); (b) bottom-to-top (called VT and VM). 


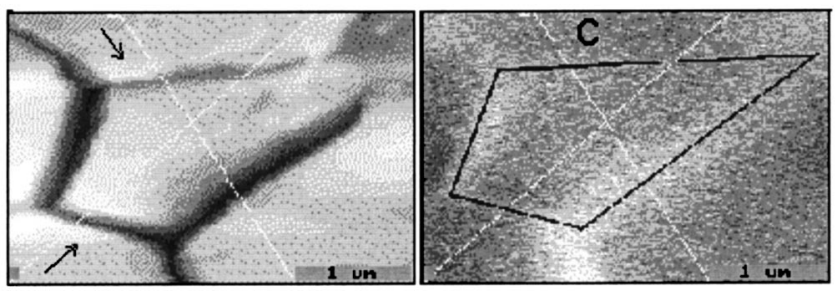

FIG. 4. Images of another single grain in sample MZ25. (Left) topographic image; (right) magnetic image. The markers have a width of $1 \mu \mathrm{m}$. The white lines show the cross sections given in Fig. 5 (with directions as indicated by the arrows). The cross sections have one grain boundary in common, indicated with $C$. The outline of the grain in the magnetic image is shown in black.

On a total of 14 grains imaged for this sample, most showed the same featureless magnetic signal inside the grains. We interpret this as the signature of a single magnetic domain. On the other hand, effects of the grain boundaries are not always absent, as demonstrated in Figs. 4 and 5. The grain displayed in Fig. 4 has a very elongated shape, with a long axis larger than $3 \mu \mathrm{m}$ and a short axis of about $1 \mu \mathrm{m}$. Cross sections along both axes are given in Fig. 5, from lower-left to upper-right [long axis, Fig. 5(a)] and from upper-left to lower-right [short axis, Fig. 5(b)]. They clearly demonstrate significant variations in magnetic signal near the
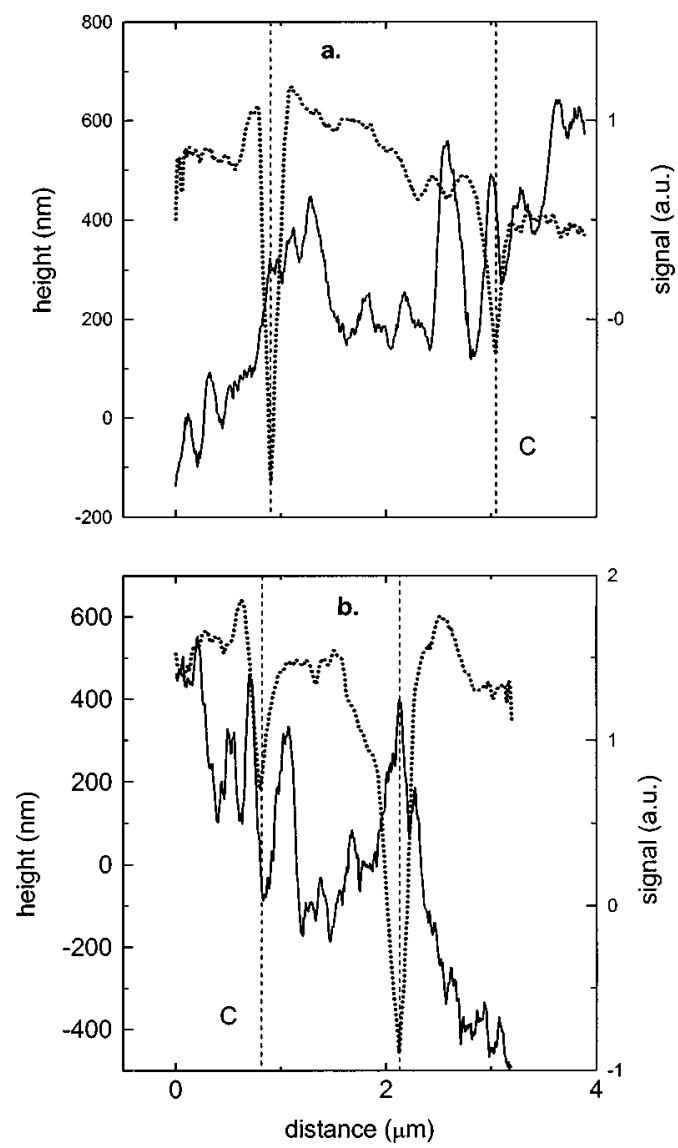

FIG. 5. Topographic (dotted line; left-hand scale) and magnetic (drawn line; right hand scale) signal variations along the cross-sectional lines given in Fig. 4. The dashed vertical lines indicate the grain boundaries (a) Lower left to upper right (long axis). (b) Upper left to lower right (short axis). The common grain boundary in both cross sections is marked $C$.

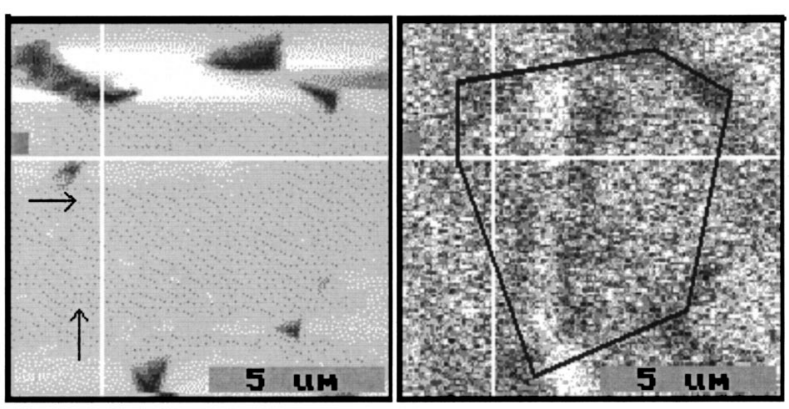

FIG. 6. Images of a single grain in sample MZ73, with an average grain size of $7.3 \mu \mathrm{m}$. (Left) topographic image; (right) magnetic image. The markers have a width of $5 \mu \mathrm{m}$. The white lines show the cross sections given in Fig. 7 (with directions as indicated by the arrows). The outline of the grain in the magnetic image is shown in black.

grain boundaries. Interestingly, the onset of the "magnetic" boundary usually starts well inside the onset of the topographic boundary. We will discuss this observation at the end of this section. Also, the two cross sections have one grain boundary in common (denoted " $C$ "' in Figs. 4 and 5), and it should be noticed that the magnetic signal variations at this boundary are very similar (and strongly peaked).

Next we discuss the sample with the largest grains, MZ73. Topographically, this sample is completely different. It is very flat, without the height differences which conveniently indicated the perimeter of the grains in MZ25. The only distinguishing features are pores, which are found regularly at the so-called triple points. The connecting lines between the pores gives a reasonable indication for the position of the grain boundaries. Figure 6 shows topographic and magnetic images of a grain of about $8 \mu \mathrm{m}$ diameter; the grain perimeters drawn in the magnetic image was produced by connecting the pores, visible as black dots in the topographic image. In the magnetic image [Fig. 6(b)], a clear feature can be seen running from top to bottom inside the grain. Left-to-right and bottom-to-top cross sections (again called HT, HM, and VT, VM, respectively) are given in Fig. 7. Starting with HT, HM [Fig. 7(a)], a pore at the left hand grain boundary is clearly visible in both images, followed by the unusually strong peak of the earlier mentioned feature in HM (indicated with an arrow), at a position where HT is completely flat. Further along the line, there is more magnetic signal variation at the position which roughly corresponds to the right hand grain boundary. We interpret the strong peak as the signature of a domain wall. The VM cross section does not show features inside the grain, consistent with the fact that it runs parallel to the feature inside the grain in the magnetic image [see Fig. 6(b)]. The trace shows signs of both grain boundaries, particularly near the deep pore at the top of the line, which again exerts its magnetic influence over a few microns.

The peaked signal variation at the domain wall position witnessed here is not the only type of variation observed. Figure 8 shows another grain of about the same size, with reasonably visible outlines from height contrast, even though the surface is rather dusty. The corresponding magnetic image shows a strong contrast change along a line roughly through the middle of the grain. A cross section (lower left to 

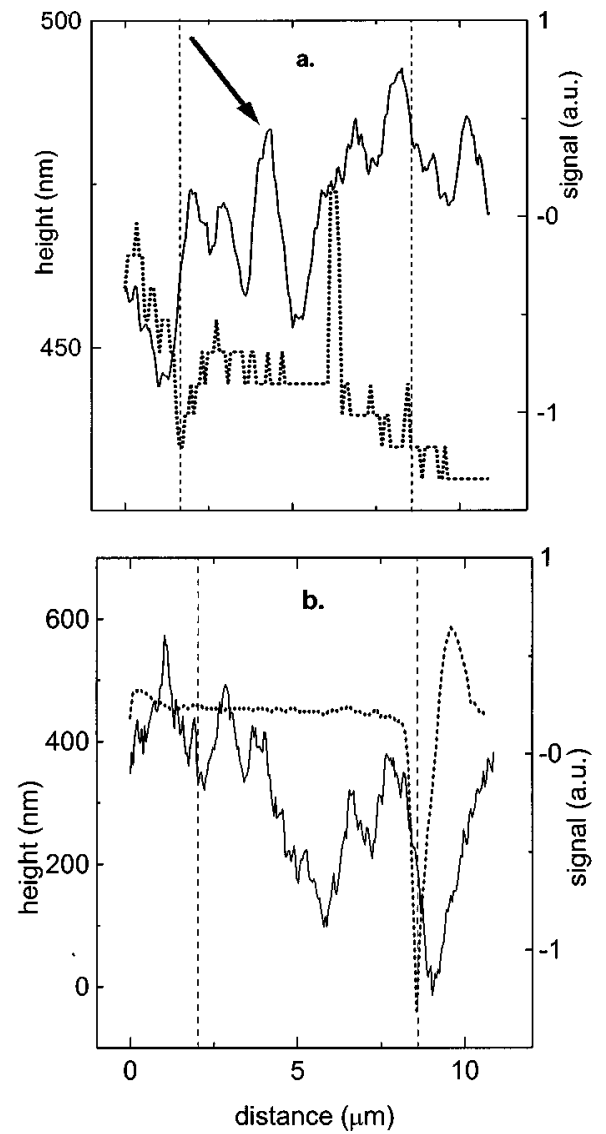

FIG. 7. Topographic (dotted line; left-hand scale) and magnetic (drawn line; right hand scale) signal variations along the cross-sectional lines given in Fig. 6. The dashed vertical lines indicate the grain boundaries. (a) Left-toright; the solid arrow indicates the domain wall feature. (b) Bottom-to-top.

upper right) is given in Fig. 9, showing the contrast change as a step in signal level. Again we interpret this feature as a domain wall, leaving the discussion of the reason for the observation of two different types of contrast to the Appendix. Ten grains were imaged, all showing a domain wall.

A description of the results of the final two samples, MZ31 and MZ54, can be brief. Both samples contain grains with a flat "monodomain" behavior as found in MZ25, as well as grains with either the peak contrast or the step contrast associated with two domains. For MZ31, a total number

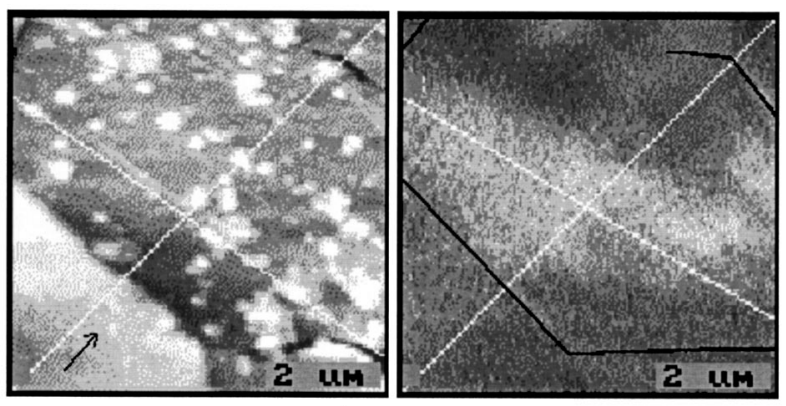

FIG. 8. Images of another single grain in sample MZ73, with an average grain size of $7.3 \mu \mathrm{m}$. (Left) topographic image; (right) magnetic image. The markers have a width of $2 \mu \mathrm{m}$. The white line shows the cross section given in Fig. 9 (with direction as indicated by the arrow). The outline of the grain in the magnetic image is shown in black.

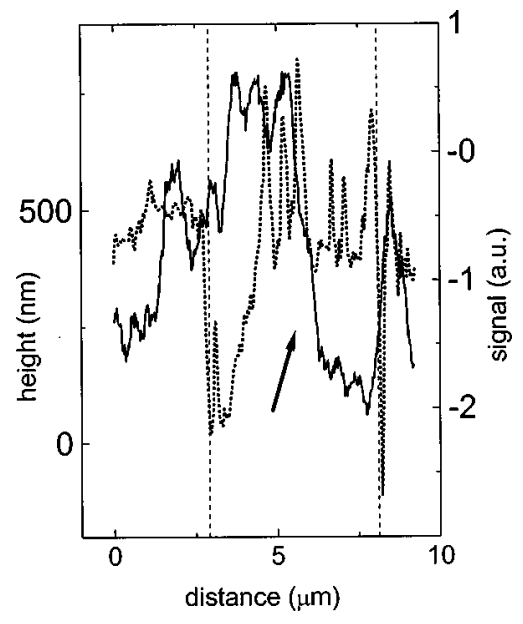

FIG. 9. Topographic (dotted line; left-hand scale) and magnetic (drawn line; right hand scale) signal variations along the cross-sectional line given in Fig. 8 . The dashed vertical lines indicate the grain boundaries. The solid arrow indicates the domain wall feature.

of 13 grains was imaged, with nine showing one domain, and four showing two domains. For MZ54, the situation is just opposite, with four grains showing one domain and nine showing two domains on a total of 13 grains imaged. Figure 10 summarizes the findings for all samples. For ease of comparison, part of the ND data for the MnZn ferrites ${ }^{4}$ are reproduced as well.

Having identified different domains within a single grain, we can also estimate the width $\delta_{m}$ of the domain walls from the images and compare these to the theoretical values, as an additional check on the interpretation of the data. Both the width of the peak in Fig. 7 and of the step in Fig. 9 are of the order of 1 to $1.5 \mu \mathrm{m}$, well above the resolution of the MFM of about $0.3 \mu \mathrm{m}$. Such a number agrees very reasonably with a simple theoretical estimate for $\delta_{m}$ from the
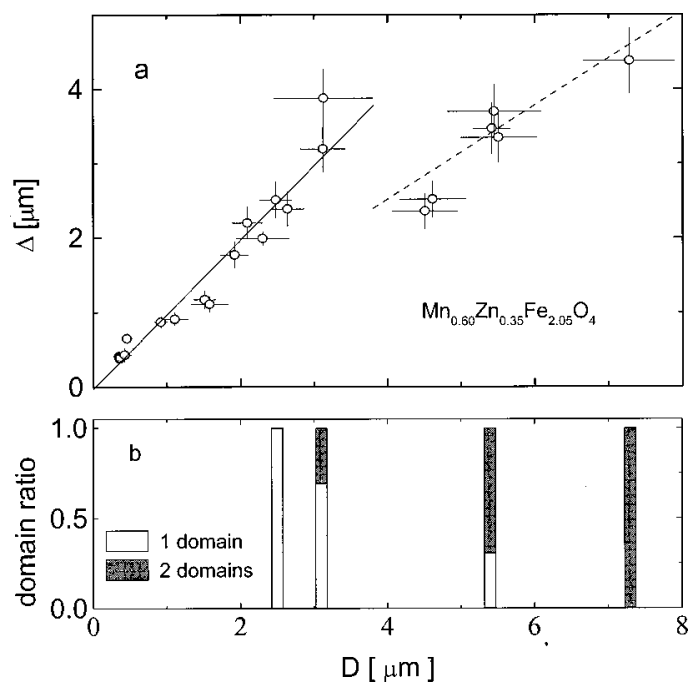

FIG. 10. (a) Domain size $\Delta$ vs grain size $D$ as determined from neutron depolarization experiments (data taken from Ref. 4). For the solid line, $\Delta / D=1$, the ratio corresponding to monodomain grains; the dashed line corresponds to $\Delta / D=0.63$, the ratio calculated for grains in the two-domain state. (b) Bar diagram showing the relative occurrence of one domain or two domains per grain for the four samples studied by MFM at their respective values for $D$. 
known material parameters of the system: $\delta_{m} \approx \sqrt{A / K}$, with $A$ the exchange constant and $K$ the anisotropy constant. Using the values $A=30 \times 10^{-12} \mathrm{~J} / \mathrm{m}$ and $K=32 \mathrm{~J} / \mathrm{m}^{3}$ (Ref. 4) we find $\delta_{m} \approx 1.3 \mu \mathrm{m}$. In an independent experiment with a different and more sensitive setup (using a tip made magnetically sensitive by electron beam deposition), ${ }^{17,18}$ we found for a NiZn ferrite a much smaller value of $\delta_{m}$ (of the order of $0.2 \mu \mathrm{m}$ ), reasonably consistent with a ten times smaller $A$ and a ten times larger $K$ for this ferrite. ${ }^{5}$ In view of the value of $\delta_{m}$ for the $\mathrm{MnZn}$ ferrite, it is also significant that the onset of the magnetic signal near the grain boundary in smallgrained samples (see Figs. 4 and 5) is found well within the grain, at a distance of more than $0.5 \mu \mathrm{m}$ from the boundary. This can be interpreted as the manifestation of a domain wall positioned at the grain boundary, extending inwards over such a long distance due to the small values of the anisotropy constant $K$. Note that this yet again shows that the grains should not be viewed as isolated particles.

Considering the overall results given in Fig. 10 and the estimates for $\delta_{m}$, the experimental picture seems straightforward. Although being based on measurements on the surface of the sample, it confirms that domain walls appear beyond a certain grain size. Grains with large diameters contain a domain wall, which more or less divides the grain in two parts. A critical grain size $D_{\text {cr }}$ determines whether a grain will accommodate one or two magnetic domains. The value of $D_{\text {cr }}$ lies around $4 \mu \mathrm{m}$; as was also found in the neutron depolarization experiments. ${ }^{3}$ Due to the distribution of grain sizes, samples with average size near $D_{\text {cr }}$ contain both grains with one and with two domains, as would be intuitively expected, making the transition from one-domain samples to two-domain samples a gradual rather than a sudden one. It is tempting to attribute the much sharper transition seen in neutron depolarization and high frequency dissipation to cooperative effects in the bulk of the sample. Moreover, the data show that in almost all cases a magnetic signal variation is present at the grain boundary, indicating that the direction of the magnetization changes from grain to grain. Especially for the small grains, it was suggested ${ }^{19}$ that grains would contain one intragranular domain wall while the magnetization would be homogeneous over the boundary, which would lead to an effective domain size equal to the mean grain size. This situation is not observed.

\section{CONCLUSIONS}

Domain walls have been observed in the surface grains of polycrystalline MnZn-ferrite samples by magnetic force microscopy. Importantly, the existence of a mono-domain state for such MnZn ferrites with grain size smaller than 4 $\mu \mathrm{m}$ is confirmed at the level of single grains. Small grains do not necessarily have a homogeneous magnetization. When a domain wall is present at the grain boundary, it extends into the grain to a considerable length. This is a logical consequence of the weak magneto-crystalline anisotropy of the material. In the surface grains under observation, the transition of the one-domain situation to the two-domain situation is gradual, following the distribution of grain sizes in the sample.

\section{ACKNOWLEDGMENTS}

The authors are indebted to A. Noordermeer for preparing the samples used in this study. Furthermore, the authors would like to acknowledge the contributions of several students of the "Technische Hogeschool Rijswijk" in building the MFM, as well as the advice of A. P. Volodin, M. Marchevsky, and H. W. van Kesteren. The authors thank A. Aharoni and the late J. P. Jakubovics for communicating the results of their micromagnetic calculations prior to publication, and to M. Guyot for stimulating discussions concerning possible domain configurations in ferrites. One of us (J.A.) wishes to acknowledge the hospitality of the University of Basel, where preparation of the manuscript was begun. The work was part of the research program of the "Stichting Technische Wetenschappen", which is part of the Netherlands Foundation for Research NWO.

\section{APPENDIX: REMARKS ON THE MAGNETIC CONTRAST}

With respect to the origin of the two different types of contrast observed in the large grains, we can make the following observations. In principle, the contrast depends on the full sample stray field and on the shape and direction of the magnetic domain in the tip which acts as sensor. As first discussed by Schönenberger and Alvarado ${ }^{20}$ and more recently by Hug et al. ${ }^{18}$ different contrast regimes can be distinguished, depending on the length of the sensor domain in the tip $L_{\text {sens }}$ and the characteristic wavelength $\lambda_{m}$ of the magnetic features of the sample. Roughly speaking, when $L_{\text {sens }}$ is smaller than $\lambda_{m}$, the tip acts as a dipole and the force on the tip is due to the field gradient produced by the sample; when the sensing domain is much longer than $\lambda_{m}$, the tip acts as a monopole and the force is due to a filtered version of the field itself. For our sputtered tips we expect the sensing domain to be small with respect to the magnetic features, which have a characteristic scale of $1 \mu \mathrm{m}$. The force is then due to field gradients. Since in our experiment the force gradient is measured, this is equivalent to the second derivative of the field along the direction of the tip. We therefore consider this second derivative of the stray field for two different directions of the magnetization on both sides of the domain wall. The stray field from the sample $\vec{H}_{s}$ is calculated by integrating over the magnetization of the sample as described by Rugar et al. ${ }^{21} \vec{H}_{s}$ is then used to calculate the force derivative in the $z$ direction, taken along the tip and perpendicular to the scan plane, on a point dipole of arbitrary direction $\mathbf{m}$ :

$$
F_{z}^{\prime}=m_{x} \frac{\partial^{2} H_{x}}{\partial z^{2}}+m_{y} \frac{\partial^{2} H_{y}}{\partial z^{2}}+m_{z} \frac{\delta^{2} H_{z}}{\partial z^{2}} .
$$

The assumption made here is that the deflection of the triangular cantilever is only due to the force along $\hat{z}$. The small misalignment of the tip with respect to the normal to the sample plane (less than $0.2 \mathrm{rad}$ ) is therefore neglected. Knowing that the magnetization for these soft magnets will lie in-plane for these (bulk) samples, the simplest model for a ferrite grain with a domain wall is a cube [see Fig. A1(a)], with its upper plane the scan plane $(z=0)$, and a domain wall represented by a step in the magnetization from a con- 

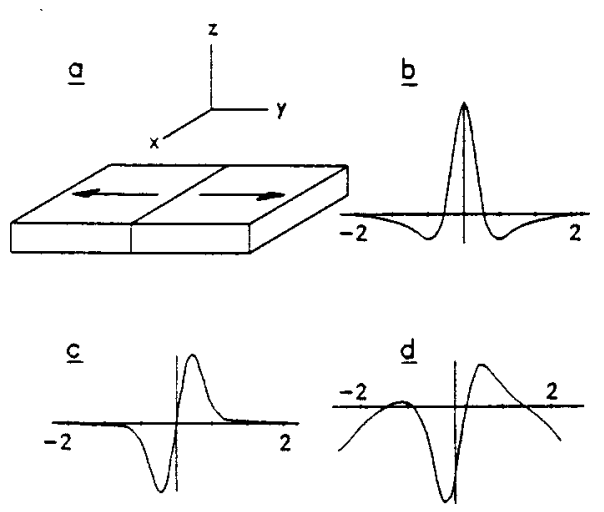

FIG. A1. (a) Model configuration for an isolated grain with a grain boundary and head-tail directions of the magnetization; force derivative $F^{\prime}$ along $x=0$ for a tip with sensing dipole (b) along the $\hat{z}$ direction; (c) along the $\hat{x}$ direction; (d) tilted $45^{\circ}$ in the $\hat{z}-\hat{x}$ plane.

stant positive to a constant negative value at the $x=0$ plane. Two choices are possible for the direction of the magnetization, along $\hat{x}$ or $\hat{y}$, and both will be considered below. Furthermore, we pay special attention to the response across the domain wall along the center line of the scan plane, for reasons to be discussed. For the direction of the magnetization along $\hat{y}$, the force derivative is well known since it is found in longitudinal bit patterns in recording tapes. The behavior along the center line is sketched in Figs. A1(b), A1(c), and A1(d) for three different directions of the sensing dipole, namely along $\hat{z}$, along $\hat{y}$ and in the $\hat{z}, \hat{y}$ plane. Obviously, a dipole along $\hat{x}$ will not generate a signal. The scan height is used to define the length scales and set to 1 , the sides of the cube are 5 times larger. For the dipole along $\hat{z}$ [Fig. A1(b)] the response is peak-like; for the dipole along $\hat{x}$ [Fig. A1(c)], $F^{\prime}$ changes sign at $x=0$, with two peaks before the signal drops to zero. The more general case of the dipole in the $\hat{z}, \hat{y}$ plane can be easily constructed from the vector sum of the given signals. For instance, tilting $45^{\circ}$ away from $\hat{x}$ still leads to a strong peak [Fig. A1(d)], with some undershoot on one side. Essentially such a configuration of magnetization and sensing dipole is probably present when the experiment shows peak-like contrast. However, the isolated cube model fails when moving away from the center line. The signals decrease towards the edges of the cube, which is not seen in the experiment. Of course, this is not surprising: the magnetic surroundings of a grain will be quite complex, precluding the simple closure of flux found in the isolated case. Comparison of the model with experiments should therefore not be attempted near the edges; equivalently, the center line response is probably representative for the full response.

Given the fact that the domain walls are quite broad so that sharp features are smeared out, and that the response will not go to zero due the stray field of the surrounding grains, it seems possible that the measured step response is also due to the configuration discussed above, with the dipole along $\hat{y}$ [Fig. A1(c)]. Another possibility to produce a step response is contained in the second choice for the magnetization directions, pointing along $+\hat{x}$ and $-\hat{x}$ on both sides of the domain wall at $y=0$ [see Fig. A2(a)]. Two directions for the sensing dipole are relevant, along $\hat{z}$ and along

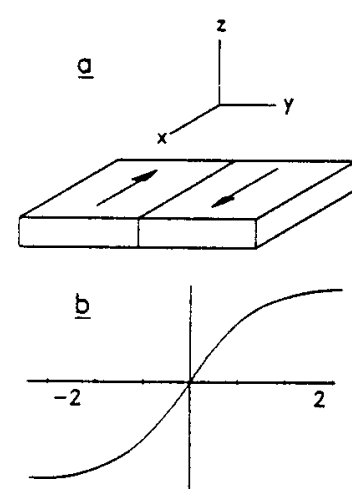

FIG. A2. (a) Model configuration for an isolated grain with a grain boundary and antiparalllel directions of the magnetization; (b) force derivative $F^{\prime}$ along $x=0$, with the sensing dipole along the $\hat{y}$ direction.

$\hat{x}$. The first case (not shown) shows zero response along both center lines of the cube $(x=0, y=0)$, and different signs of the response in all four quadrants of the measured surface. Step responses exist therefore, but they change sign when passing one of the center lines. Such behavior has not been found in the experiments. The second case [Fig. A2(b)] shows a step response, which is quite flat in the middle and very steep at the sides of the grain, again because of flux closure of the isolated cube; assuming as before that the latter will be modified by the surroundings, this configuration may explain the response found in Fig. 9. However, such a configuration will not produce a peaked response.

These elementary considerations still lead to some qualitative conclusions. One is that the grains are clearly not magnetically isolated. Although this may seem obvious, it is important in view of the fact that micromagnetic calculations fail to reproduce the critical thickness for domain wall formation, unless the magnetic environment is taken into account in some manner. The other tentative conclusion is that the peak-like domain wall contrast found in a number of grains stems from a head-tail magnetization configuration rather than an anti-parallel one, although the latter might be thought more probable. Whether or not this is only true for surface grains cannot be answered.

${ }^{1}$ E. C. Snelling, Soft Ferrites: Properties and Applications, 2nd ed. (Butterworths, London, 1988).

${ }^{2}$ D. Stoppels, J. Magn. Magn. Mater. 160, 323 (1996).

${ }^{3}$ P. J. van der Zaag, M. T. Johnson, A. Noordermeer, P. T. Por, and M. Th. Rekveldt, J. Magn. Magn. Mater. 99, L1 (1991).

${ }^{4}$ P. J. van der Zaag, J. J. M. Ruigrok, A. Noordermeer, M. H. W. M. van Delden, P. T. Por, M. Th. Rekveldt, D. M. Donnet, and J. N. Chapman, J. Appl. Phys. 74, 4085 (1993).

${ }^{5}$ P. J. van der Zaag, P. J. van der Valk, and M. Th. Rekveldt, Appl. Phys. Lett. 69, 2927 (1996).

${ }^{6}$ A. Noordermeer and M. M. E. Vantilt, in Ceramic Powder Processing Science, edited by H. Hausner, G. L. Messing, and S. Hirano (DKG, Köln, 1989), pp. 617-624; A. Noordermeer and M. M. E. Severin-Vantilt, Adv. Mater. 3, 394 (1991).

${ }^{7}$ A. Noordermeer, Ph.D. thesis, Eindhoven University of Technology (1997).

${ }^{8}$ P.J. van der Zaag, J. Magn. Magn. Mater. 196-197, 315 (1999).

${ }^{9}$ W. F. Brown, Jr., J. Appl. Phys. 39, 993 (1968).

${ }^{10} \mathrm{~S}$. Chikazumi and S. H. Charap, Physics of Magnetism (Krieger, Malabar, 1986), pp. 239-241. 
${ }^{11}$ A. Aharoni and J. P. Jakubovics, IEEE Trans. Magn. 24, 1892 (1988).

${ }^{12}$ A. Aharoni and J. P. Jakubovics, J. Phys. IV (France), 8, Pr2-389 (1998).

${ }^{13}$ C. Kittel, Phys. Rev. 70, 965 (1946).

${ }^{14}$ J. E. Knowles, Br. J. Appl. Phys., J. Phys. D 1, 987 (1968).

${ }^{15}$ Y. Martin and H. K. Wickramasinghe, J. Appl. Phys. 61, 4723 (1987).

${ }^{16}$ It is well known that differently oriented surfaces of ferrite crystals show different hardnesses and etching rates. This will lead to differences in polishing rates for differently oriented grains in the sintered ferrite.

${ }^{17}$ Courtesy of H. J. Hug, P. van Schendel, and H.-J Güntherodt, Basel University.

${ }^{18}$ H. J. Hug et al., J. Appl. Phys. 83, 5609 (1998).

${ }^{19} \mathrm{M}$. Guyot and P. Perriat (private communication).

${ }^{20}$ C. Schönenberger and S. F. Alvarado, Z. Phys. B 80, 373 (1990).

${ }^{21}$ D. Rugar, H. J. Mamin, P. Guethner, S. E. Lambert, J. E. Stern, I. McFadyen, and T. Yogi, J. Appl. Phys. 68, 1169 (1990). 